\title{
The Use of Virtual Reality in Motor Learning: A Multiple Pilot Study Review
}

\author{
Einat Yanovich, Omri Ronen \\ The Zinman College of Physical Education and Sport Sciences, Wingate Institute, Netanya, Israel \\ Email: einaty@wincol.ac.il
}

Received 8 July 2015; accepted 3 August 2015; published 6 August 2015

Copyright (C) 2015 by authors and Scientific Research Publishing Inc.

This work is licensed under the Creative Commons Attribution International License (CC BY). http://creativecommons.org/licenses/by/4.0/

(c) () Open Access

\begin{abstract}
In recent years, technological improvements have allowed for the creation of V.R. environments for different uses, especially in the training of pilots, astronauts, medical staff, soldiers, and athletes. In regards to physical activity, V.R. is currently being used in two main fields: Exergaming and Rehabilitation. The purpose of this article is to investigate the use of this technology as a means of demonstrating and learning motor abilities in many types of populations and situations. Three studies were done using V.R. In all three of them healthy participants were assigned to a control or test group. These studies were done using two main V.R. systems designed for motor learning: Timocco and IREX. Study 1 tested bi-lateral transfer in the upper limbs; Study 2 tested the differences in improvement using V.R. between internal and extrinsic focus of attention; Study 3 tested differences in different learning strategies in motor tasks-massed practice vs. distributed practice. Study 1 found significant differences between control and test groups; Study 2 did not find that external focus of attention was superior as expected but found a stronger correlation between tests at different days; Study 3 found no significant improvements $(p>0.05)$ for each group. In conclusion, V.R. can be an effective means of teaching and training basic motor skills, sometimes even superior to "real-life" because of the highly modifiable environment and difficulty in the comfort of one's clinic or home.
\end{abstract}

\section{Keywords}

Virtual Reality, Motor Learning, Timmoco, IREX

\section{Introduction}

Virtual reality (V.R.), sometimes referred to as immersive multimedia, is a computer-simulated environment that can simulate physical presence in places in the real world or imagined worlds. The user controls his/her actions in the V.R. by a remote input device, such as a keyboard or a mouse, or by an advanced apparatus such as a camera, special gloves, or other objects. 
In recent years, technological improvements have allowed for the creation of V.R. environments for different uses, especially in the training of pilots, astronauts, medical staff, soldiers, and athletes. In regards to physical activity, V.R. is currently being used in two main fields: Exergaming and Rehabilitation. The purpose of this article is to investigate the use of this technology as a means of demonstrating and learning motor abilities in many types of populations and situations.

In the past few years, new V.R. technology is being developed and purchased by major technology companies, in investments of millions and even billions of dollars. This trend shows a vote of confidence by these companies in the importance and application of these new technologies in different areas and scenarios.

As a result, hundreds of articles are being published on this topic each year. These articles study a variety of fields, ranging from improvements in motor abilities to the psychological benefits of V.R.

Wille et al. (2009) previously conducted a pilot study involving a system for rehabilitation named "PITS" (Paediatric Interactive Therapy System) and found that "the results of the pilot study suggest that PITS is a feasible, applicable and motivating V.R. system which can be applied in rehabilitation settings for training in children with congenital or acquired motor dysfunctions of the upper limbs" (Wille et al., 2009: p. 44).

Chen, Jeng, Fung, Doong, \& Chuang (2009) investigated the psychological benefits of virtual reality in rehabilitation in regards to the patient's tension, and found that "a virtual-reality-based rehabilitation program can ease patients' tension and induce calm" (Chen et al., 2009: p. 258).

A systematic review published by Sandlund and colleagues (Sandlund, Medonough, \& Hager-Ross, 2009) reviewed 16 articles that investigated movement quality, spatial orientation and mobility, and motivational aspects in children with sensorimotor disorders, and found that 13 of the articles had positive results, although two of the three randomized control trials found no significant improvements in spatial orientation.

In the past few years, assessment studies have been completed at the Zinman College of Physical Education and Sport Sciences on a young healthy population (physical education students) in order to examine the possible applications suggested in the area of motor learning.

These studies were done using two main V.R. systems designed for motor learning: Timocco and IREX.

Timocco is an interactive and motion based computer game for children with difficulties in motor control and cognitive skills, used in clinics, schools, and at home. As part of occupational therapy or physiotherapy adapted to each special need, motor and cognitive skills improve in a fun, safe and non-violent gaming environment. Each game focuses on specific abilities, and enables the user to select between levels of difficulty and the games can be adopted to the child's specific needs. Performance results are saved in a database on the Internet or on a local network, and can be viewed and analyzed any time.

Gesture Tek's Interactive Rehabilitation and Exercise System $\left(\right.$ IREX $\left.^{\circledR}\right)$ uses immersive video gesture control technology to place patients into virtual sport or gaming environments, where they are guided through clinicianprescribed therapeutic exercise regimes. Clinicians can design a fun and interactive exercise program that tests single joints, combined joint movements, or full body function.

The assessment studies tested major motor learning strategies such as internal vs. extrinsic focus of attention, massed vs. distributed practice, and bi-lateral transfer.

In order to assess the potential of V.R., the purpose of these studies was to validate the results from V.R. training in "real-life" training results from the literature.

A study conducted by Teixeira (2000) on bi-lateral transfer tested both fine force and a simple anticipatory timing task in two separate experiments, and found that bilateral transfer of learning took place for both anticipatory timing and force control, and significant transfer only in the preferred to non-preferred direction in force control.

In 2001, Wulf, Shea, and Park performed a 3-day experiment and asked the participants to choose internal or external focus of attention. On the third day most of the participants chose an external focus, and were more effective in retention than participants who preferred an internal focus. Wulf co-authored several other studies (e.g., Shea \& Wulf, 1999; Wulf, Lauterbach, \& Tool, 1999; Wulf, Hoss, \& Prinz, 1998; Zachry, Wulf, Mercer, \& Bezodis, 2005) and found similar results for the advantages of external focus in all of them.

A meta-analytic review published in 1999 by Donovan and Radosevich comparing massed practice and spaced (or distributed) practice indicated that individuals in spaced practice conditions performed significantly higher than those in massed practice conditions. Subsequent analyses, however, suggested that the nature of the task being practiced - the inter-trial time interval, and the interaction between these two variables, significantly moderated the relationship between practice conditions and performance. Also, Donovan \& Radosevich 1999) found that the effect size was higher in studies with low methodological rigor as compared with those studies 
higher in rigor.

\section{Method}

Three studies were done using V.R. In all three of them healthy participants were assigned to one of two groups. They did not receive prior information on the purpose of the study or any information about or training in the system. Each participant performed the tests and the training in a room with only the tester present.

Study 1 tested the application of bi-lateral transfer in the upper limbs using the Timocco system using special gloves in front of a screen with a camera.

Eighteen healthy participants ( 6 male and 12 female, mean age 24) were randomly assigned to a test or a control group. The test included sitting on a chair and "blowing up" balloons with their dominant hand using one of two special gloves (one red and one green) according to the "pin" (the pin was either red or green) showing up on the screen.

Each participant had to blow up 10 balloons, and the game was set to the highest setting.

The study included three sessions: the first session included an explanation on the task and a trial followed by a test. The control group did a non-related motor task (kicking a rubber ball with two feet while sitting) while the test group received training on the specific task using their dominant limb. In the second session the test group received additional training while the control group did only the non-related motor task.

In the third session the test group performed additional training on the system their dominant limb, and then proceeded to the post test followed by the transfer test that involved using the non-dominant hand in same test, While the control group did the non-related motor task followed by the post test (same setting as the pre test) and then did the Transfer test (same setting as the pre test). All post tests were done using only the non-dominant hand.

Study 2 tested the differences in improvement using V.R. between internal and extrinsic focus of attention using the Timocco system, using special gloves in front of a screen with a camera. Twenty healthy participants (10 male and 10 female, mean age 25.5), were randomly assigned to an internal focus of attention group or an extrinsic focus of attention group. Each group consisted of 5 males and 5 females. The study was performed in four sessions of 60 seconds. The internal group was told to focus their attention to their own body parts movement, while the extrinsic group was told to focus their attention to the moving objects on the screen. The first session was a pre test done while sitting on a chair in front of a screen wearing special gloves. The purpose was to "bounce" aliens. All of the aliens were the same kind, the pace at which they were advancing was medium, were small-sized, were far away at set locations, and were given numbers. The game had background and sounds. The purpose is to bounce as many aliens as possible in 60 seconds while not touching the alien with the specific number of the spaceship at the top of the screen. The second and third sessions were done two days later. The second was the same as the first session and the third included a new difficulty-the aliens changed their locations. The last session was performed a week later and was the same as the third, but included additional types of aliens.

Study 3 tested differences in different learning strategies in motor tasks-massed practice vs. distributed practice, using the IREX system using special gloves in front of a screen with a camera. Sixteen healthy participants (6 male and 10 female, mean age 26), were randomly assigned to one of the groups (massed or distributed).The study was done in 5 sessions, taking place on two separate days. The purpose of the game was to juggle balls. The first session included 2 trials of 45 seconds at level 5. Based on the success rate of these trials the participants were assigned to one of the two groups, in order to create equal groups at the baseline. In the second session the massed practice group preformed 7 sets of 45 seconds, separated by 15 seconds of rest at level 5 . The Distributed Practice group preformed 7 sets of 45 seconds separated by 15 seconds of rest followed by 30 seconds of "snowboard" practice and 15 second of rest at level 5. On day two the massed practice group performed the same protocol of training as on day one, then the group preformed 2 trials of 45 seconds at level 5 and a test of 2 trials of 45 seconds at level 7.

Participants in the Distributed Practice group preformed the same protocol of training as day one. Then the group preformed 2 trials of 45 seconds at level 5 and a test of 2 trials of 45 seconds at level 7.

\section{Results}

The first study tested parameters of completion time, reaction time, and the correlation between post and transfer 
tests in both of these parameters.

Completion time $($ control $=218.67 \pm 19.23$, test $=223.56 \pm 21.51$, Figure 1$)$ and reaction time $($ control $=1.70$ \pm 0.33 , test $=1.87 \pm 0.39$, Figure 2$)$ in the pre test showed no differences in either of these parameters between the groups $(p>0.05)$. In the post test, parameters of completion time (control $=232.0 \pm 27.92$, test $=193.89 \pm$ 13.36) and reaction time (control $=1.77 \pm 0.42$, test $=1.19 \pm 0.16)$ were significantly $(p<0.01)$ better in the test group. The test group significantly improved Reaction time $(p<0.01)$; no significant improvement was found in the completion time $(p>0.05)$.

The parameter tested in the second study was the score in the tests. No significant differences were found between the groups in the pre test (external $=249 \pm 192.21$, internal $=227 \pm 87.06, p>0.05$, Figure 3). No significant differences were found between the groups in the acquisition test (external $=397 \pm 240.37$, internal $=$ $258 \pm 83.77, p>0.05)$.

In the transfer test, the internal focus group achieved a higher score than in the retention test (retention $=229$ \pm 61.9 , transfer $=235 \pm 35.36$ ), but a Pearson correlation between the test was only $r=0.49$. The external focus group showed a similar trend (retention $=398 \pm 207.89$, transfer $=495 \pm 226.83)$, but with a strong correlation $(\mathrm{r}$ $=0.86$ ). Pearson correlation between the retention test and the transfer test in the Extrinsic focus group were strongly correlated $(\mathrm{r}=0.86)$, while the results of the internal focus did not show similar results $(\mathrm{r}=0.49)$.

In the third study, which tested massed vs. distributed practice (Figure 4), no significant improvements ( $p>$ 0.05 ) were found for either group between pre (massed $119.25 \pm 18.14$, distributed $119.38 \pm 19.13$ ) and post (massed $124 \pm 30.18$, distributed $127.25 \pm 14.93$ ).

Pearson correlation between post and transfer tests found that the achievements of both the distributed practice group and massed practice group were strongly correlated (distributed $=0.82$, massed $=0.87$ ).

\section{Discussion}

The results from the pilot study on bi-lateral transfer correlated with the literature showing that dominant limb training can increase performance in the non-dominant limb even without training. The second study did not

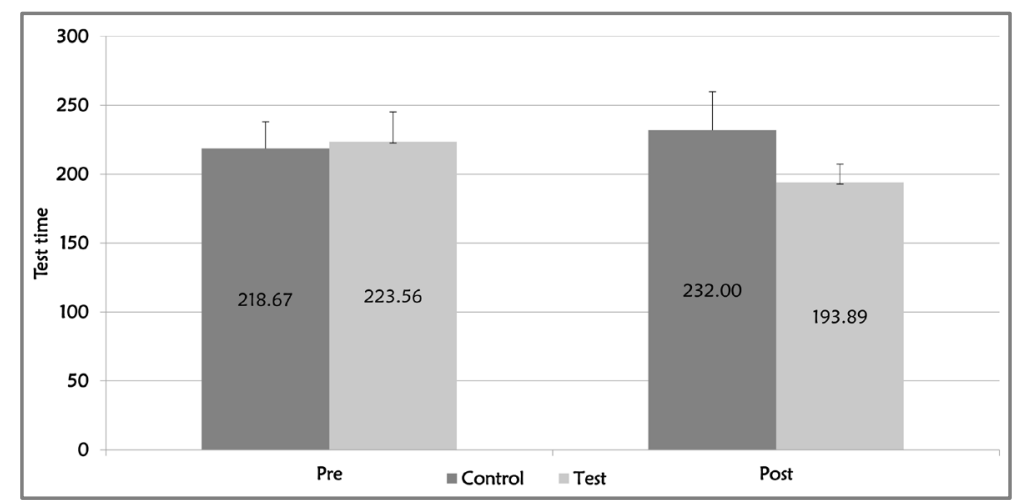

Figure 1. Bi-lateral transfer completion time.

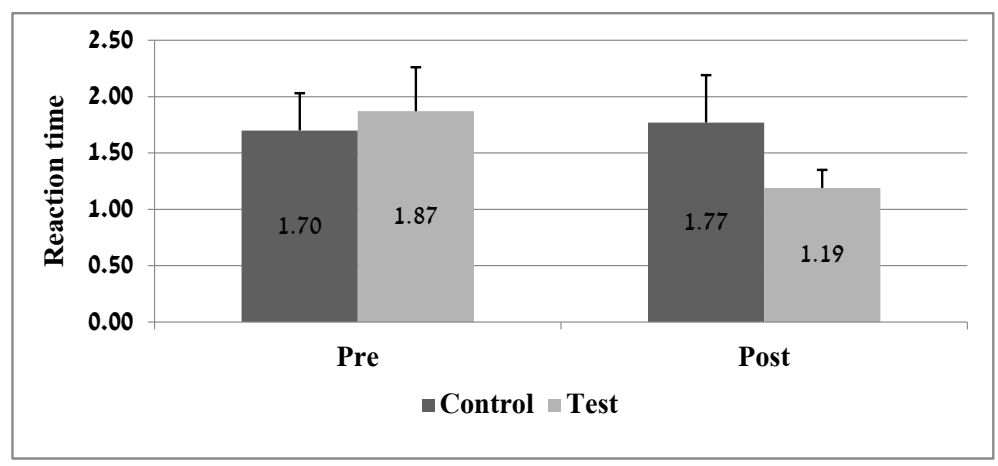

Figure 2. Bi-lateral transfer reaction time. 


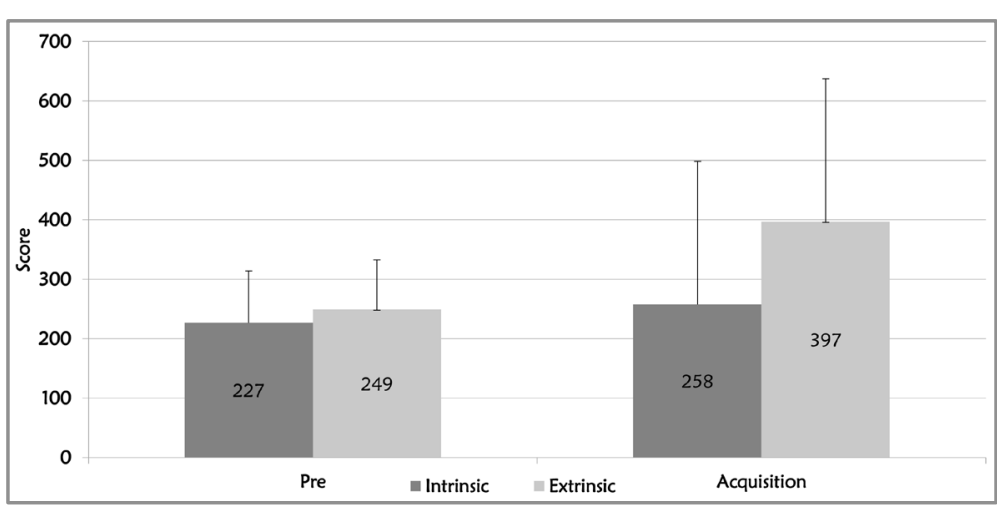

Figure 3. Internal vs. external focus of attention.

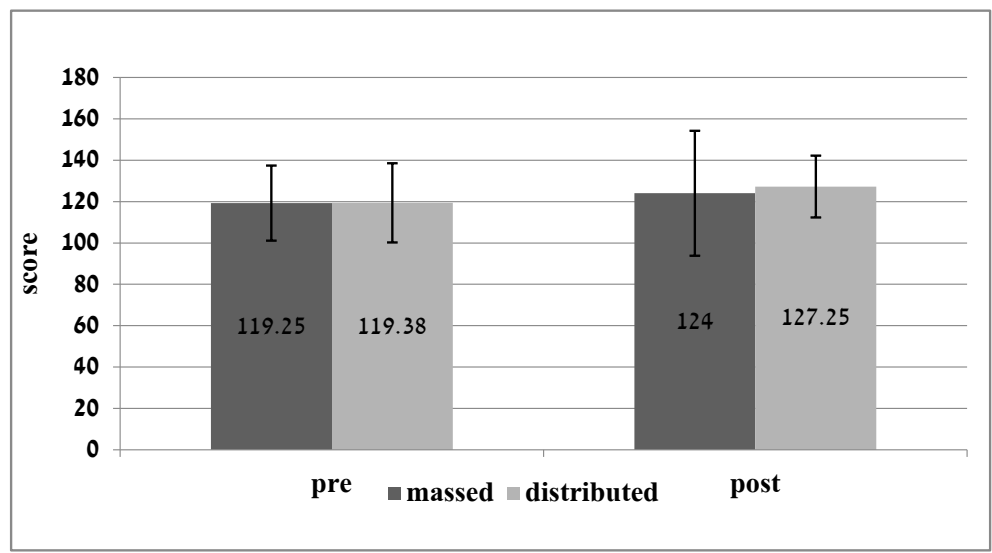

Figure 4. Massed vs. distributed practice.

show any significant difference between the groups in the acquisition test, although there was a higher average score in the test group; the retention test and transfer test was found to be strongly correlated, meaning that the external focus helped maintain the same level of performance.

While the results did not show a significant advantage for the external focus, perhaps because the system tested was designed for children and therefore the difficulty level was too easy, the trend towards the external focus group and the maintenance of performance level corresponds with the findings of Wulf and his colleagues (Wulf, Shea, \& Park, 2001; Shea \& Wulf, 1999; Wulf, Lauterbach, \& Toole, 1999; Wulf, Hoss, \& Prinz, 1998; Zachry, Wulf, Mercer, \& Bezodis, 2005).

The last study, which tested massed vs. distributed practice, showed that both of the strategies are efficient for increasing performance. Like Donovan \& Radosevich (1999), we conclude that while distributed practice is supposed to be superior, this is dependent on many variables, and we found only a slight difference between the two strategies.

Since the findings of these pilot studies are in accordance to findings from tests done in real life scenarios, it is safe to assume that V.R. training of motor skills using these systems can be equal to training in "real-life" scenarios. With the addition of the highly modifiable environment and difficulty in the comfort of one's clinic or home, V.R. can even be superior to real-life environments in some aspects.

\section{Conclusion}

We conclude that V.R. can be an effective means of teaching and training basic motor skills, and that the use of V.R. in the area of motor learning can be of great benefit in many situations and in more and more types of populations. It will be interesting to perform additional studies on these systems, like those using a V.R. headset that simulates a more realistic world. In addition, further studies on specific populations, such as children with DCD (developmental coordination disorder), should be planned in the near future. 


\section{References}

Chen, C. H., Jeng, M. C., Fung, C. P., Doong, J. L., \& Chuang, T. Y. (2009). Psychological Benefits of Virtual Reality for Patients in Rehabilitation Therapy. Journal of Sport Rehabilitation, 18, 258.

Donovan, J. J., \& Radosevich, D. J. (1999). A Meta-Analytic Review of the Distribution of Practice Effect: Now You See It, Now You Don't. Journal of Applied Psychology, 84, 795. http://dx.doi.org/10.1037/0021-9010.84.5.795

Sandlund, M., Mcdonough, S., \& Hager-Ross, C. (2009). Interactive Computer Play in Rehabilitation of Children with Sensorimotor Disorders: A Systematic Review. Developmental Medicine \& Child Neurology, 51, 173-179. http://dx.doi.org/10.1111/j.1469-8749.2008.03184.x

Shea, C. H., \& Wulf, G. (1999). Enhancing Motor Learning through External-Focus Instructions and Feedback. Human Movement Science, 18, 553-571. http://dx.doi.org/10.1016/S0167-9457(99)00031-7

Teixeira, L. A. (2000). Timing and Force Components in Bilateral Transfer of Learning. Brain and Cognition, 44, 455-469. http://dx.doi.org/10.1006/brcg.1999.1205

Wille, D., Eng, K., Holper, L., Chevrier, E., Hauser, Y., Kiper, D., \& Meyer-Heim, A. (2009). Virtual Reality-Based Paediatric Interactive Therapy System (PITS) for Improvement of Arm and Hand Function in Children with Motor Impairment-A Pilot Study. Developmental Neurorehabilitation, 12, 44-52. http://dx.doi.org/10.1080/17518420902773117

Wulf, G., Hoss, M., \& Prinz, W. (1998). Instructions for Motor Learning: Differential Effects of Internal versus External Focus of Attention. Journal of Motor Behavior, 30, 169-179. http://dx.doi.org/10.1080/00222899809601334

Wulf, G., Lauterbach, B., \& Toole, T. (1999). The Learning Advantages of an External Focus of Attention in Golf. Research Quarterly for Exercise and Sport, 70, 120-126. http://dx.doi.org/10.1080/02701367.1999.10608029

Wulf, G., Shea, C., \& Park, J. H. (2001). Attention and Motor Performance: Preferences for and Advantages of an External Focus. Research Quarterly for Exercise and Sport, 72, 335-344. http://dx.doi.org/10.1080/02701367.2001.10608970

Zachry, T., Wulf, G., Mercer, J., \& Bezodis, N. (2005). Increased Movement Accuracy and Reduced EMG Activity as the Result of Adopting an External Focus of Attention. Brain Research Bulletin, 67, 304-309.

http://dx.doi.org/10.1016/j.brainresbull.2005.06.035 